\title{
Imagery and paired-associate recognition
}

GLEN A. RASER and WAYNE H. BARTZ. Iowa State Unilersity, Ames, lowa

Three groups of $S s(N=60)$ given Imagery, Word, or vo instructions were presented 40 paired-associates once and tested in a recognition procedure. The pairs were composed of nouns and/or their line drawings. Imagery instructions resulted in higher, word instruction lower, performance than did the control instructions. Line drawings facilitated performance as response terms, but not as stimulus terms. The results were taken as support for a differentiation effect interpretation of imagery in paired-associate learning.

Studies of paired-associate learning of nouns differing in terms of rated image-evoking capacities have shown high imagery nouns to have greater effects among stimulus terms than among response terms (Paivio, Yuille, \& Smythe, 1966; Paivio \& Madigan, 1968). This effect is contrary to that found for stimulus materials rated on other measures (e.g., meaningfulness) and has led Paivio (1965) to offer the "conceptual peg" hypothesis. This hypothesis states that high imagery stimulus terms easily evoke a sensory image which is then related to the response term and serves as a cue for retrieval of the response term. Thus concrete nouns are superior to abstract nouns (Paivio, 1965), and pictures, which provide images directly, have been shown to be superior to their verbal labels (Paivio \& Yarmey, 1966).

Dominowski \& Gadlin (1967) have suggested that, contrary to the "conceptual peg" hypothesis, imagery provides stimulus differentiation which, in turn, reduces intralist interference. It is possible to speculate that in the typical recall procedure, where stimulus terms are presented as $S$ recalls the response terms, imagery could provide a basis for stimulus differentiation. If this is the case, a recognition procedure might provide a basis for response differentiation and the reported superiority of imagery for stimulus terms might not occur.

This study was designed to investigate the role of imagery in paired-associate recognition. Differences in the imagery of stimulus and response terms of pairs was established by using words and their drawings (Paivio \& Yarmey, 1966) in all four possible pair combinations. To further investigate the role of imagery, Ss were instructed to use imagery during learning. As a comparison, other $\mathrm{Ss}$ were instructed to use the characteristics of words and others were not given specific associative instructions.

\section{SUBJECTS}

The $180 \mathrm{Ss}$ were students enrolled in psychology courses at lowa State University. Fifteen were assigned in a random fashion to each of 12 treatment combinations ( 3 Instructions by 4 List Types) and were run in a group.

\section{STIMULUS MATERIALS}

The initial item pool consisted of 160 monosyllabic nouns selected from Thorndike \& Lorge (1944) which could be represented by a simple line drawing. Each word and its line drawing was separately reproduced in black ink on a $3 \times 5$ card. forty basic word pairs were then randomly selected and $35 \mathrm{~mm}$ nigative slides were inade of each of the four pair combinations of words and pictures (Word-Word. Word-Picture, Picture-Word, and Picture-Picture). Slides for the recogmition task were prepared by photographing the stimulus term alone for each pair. The response membur of each pair was photographed in a set of seven like items. The six incorrect afternatives included two response terms and two stimulus terms from other pairs and two items randomly selected from the remuining pool of items. A complete set of the test slides was prepared trsing the printed words as was one using the line drawings.

\section{INSTRUCTIONS}

One-third of the Ss were instructed to find a unique mental image to relate members of a pair. Another third were instructed to find a word or letter cue to aid in relating the pairs. Both of these groups were given examples of the type of strategy they were to use. The remaining third were simply instructed to learn or relate the pairs.

\section{PROCEDURE}

A Kodak 800 Carousel slide projector was used to present the stimulus materials. After the group's particular instructions, the 40 pairs were presented for $8 \mathrm{sec}$ each only once. (A pilot study had shown that $15 \mathrm{sec}$ presentation time resulted in extremely high levels of recognition.) The test trial followed and consisted of presentation of the stimulus term of the pair for $2 \mathrm{sec}$ and the response alternatives for $10 \mathrm{sec}$. A -different random order was used in presenting the pairs during the test trial. $S$ was to select and record his response on a data sheet during the $10 \mathrm{sec}$ response slide interval. Ss were then asked to briefly describe, in writing, the mental image, word or letter cue, or any other method they used in learning each pair.

\section{RESULTS}

The recognition results are shown in Fig. 1. The Instructional conditions differed significantly, $F(2,168)=15.26(p<.01)$. Tests among these means showed that Imagery instructions resulted in significantly higher performance than did either of the other two $(p<.01)$ : the No instructions condition resulted in higher performance than did Word instructions $(p<.05)$. The effects of list types was also significant, $F(3,168)=7.04(p<.01)$. Tests among these means were conducted by contrasting performance for list types as a function of the types of stimulus materials used as the stimulus and response terms. These tests showed that the degree of imagery was a factor among response terms, $F(1,168)=19.82(p<.01)$; pairs with Pictures as response terms were better recognized than were those with Words. Imagery was not found to be a significant factor among stimulus terms as pairs with Words and those with Pictures as stimulus terms were recognized equally well $(F<1)$.

Figure 2 shows the types of errors made on each of the List Types in terms of per cent total errors within List Type groups. Fhe incorrect alternatives in the recognition task had been either response items, stimulus items, or words not in the lists. Stimulus and response errors were made in about the same proportion in all but the Word-Picture groups which showed a much higher incidence of sclecting response term alternatives. Groups with Words as response terms made more extra-list errors as compared to groups with Pictures as response terms. The distribution of

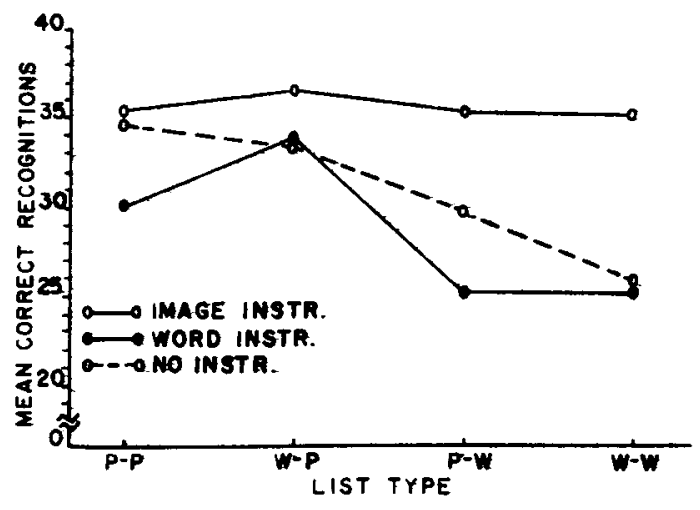

Fig. 1. Mean correct recognitions as a function of List Type and Instructions. 


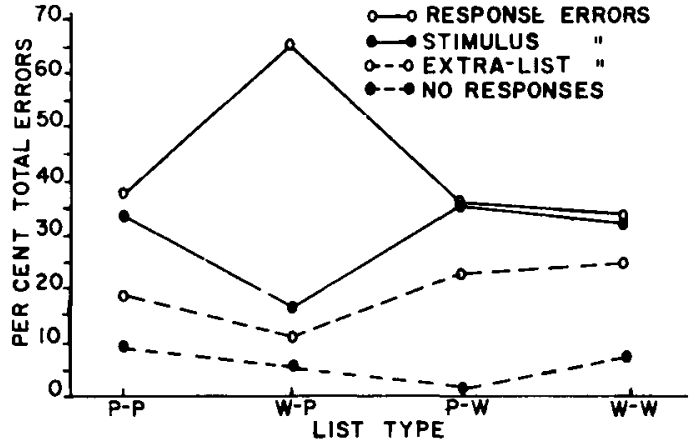

Fig. 2. Per cent of total errors for each type of error as a function of List Type.

types of errors among the Instructional conditions showed no large or consistent differences nor was there any interaction evident between Instructional conditions and List Types.

The S's written reports on how each pair was remembered were classified according to whether they represented mental imagery, word or letter cues, or other strategies. Reports were classified as mental imagery if mental "pictures" could be generated from them and as word strategies if words or parts of words were equated or combined in some way to help associate any given pair. The per cent of each type of report for each Instructional condition is shown in Fig. 3. Imagery groups reported the highest percentage of pairs on which imagery was used and Word groups the lowest. However, the Word groups did report imagery more frequently than they reported word strategies. The No Instructions groups showed percentages of imagery and word reports which were intermediate to the other groups on all three classifications.

\section{DISCUSSION}

Imagery instructions facilitated performance while word instructions hindered performance as compared to the control (No Instructions) group. Exactly this same trend was observed for the percentages of reported image mediators within instruction groups. Considering the required task and the type of stimulus materials used, these similar trends suggest a straight-forward explanation consistent with Dominowski \& Gadlin's (1967)

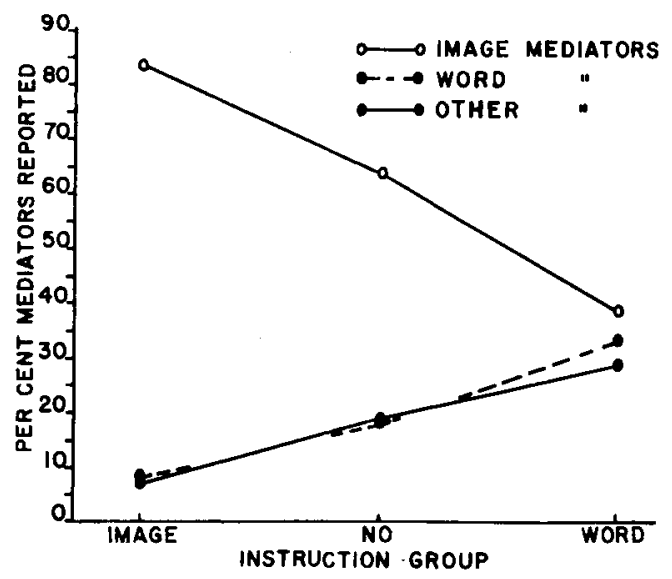

Fig. 3. Per cent mediators reported for each type of mediator as a function of Instructions. differentiation hypothesis. With highly concrete nouns and their pictorial representations the imagery strategy is efficient for it draws attention to the features which serve to discriminate them from one another. That is, the appearance of the object denoted by the nouns and pictures is criterial for discrimination. Imagery instructions thus facilitated performance, while word instructions may have served to detract from these features and thus hindered performance. It is possible that a situation could be invented where word instructions would result in higher performance than image instructions, if such features of the stimulus materials were criterial for discrimination.

The facilitating effect of pictures as response terms is opposite to predictions of the "conceptual peg" hypothesis. Since the recognition task places more emphasis on the response term, it is possible that stimulus imagery effects were obscured by the recognition procedure. Consideration of the error data however, also offers evidence opposite to the "conceptual peg" hypothesis. The analysis of errors showed that the performance of the Word-Picture groups was different from that of the others. This group selected more alternatives that had been response terms and fewer that had been stimulus terms. The other groups' stimulus and response errors were equal in number. This finding is not as clear as might be desired because of the necessary translation of the stimulus terms from one type of item to the other from presentation to recognition. Thus for stimulus terms the error data indicates that the translation of Word stimulus terms into Picture alternatives decreases the number of (stimulus) errors while the translation of Picture stimulus terms into Words does not affect the number of (stimulus) errors. Correspondingly, it seems more likely that Ss might show greater agreement on the name of a picture than they would generate the same picture for a word. The higher incidence of extra-list errors with Word responses than with Picture responses also suggests the presence of an increased discriminability among high imagery terms (Pictures) than among low imagery terms (Words).

It is possible that the "conceptual peg" hypothesis refers to one limited aspect of discriminability. If such is the case, then the "conceptual peg" in this more general frame of reference need not be imaginal or necessarily be related to concreteness. Rather, the conceptual peg or criterial discriminanda would be a function of the particular stimulus materials and the response requirements placed on S. Conceiving of the conceptual peg in this manner makes it a problem of understanding the nature of encoding stimuli rather than presupposing a particular encoding procedure.

\section{REFERENCES}

DOMINOWSKI, R. L., \& GADLIN, H. Learning as a function of stimulus-aroused imagery. Paper read at the Psychonomic Society Convention, Chicago, 1967.

PAIVIO, A. Abstractness, imagery and meaningfulness in paired-associate learning. Journal of Verbal Learning \& Verbal Behavior, 1965, 4, 32-38.

PAIVIO, A., \& MADIGAN, S. A. Imagery and association value in paired-associate learning. Journal of Experimental Psychology, 1968, 76, 35-39.

PAIVIO, A., \& YARMEY, A. D. Pictures vs words as stimuli and responses in paired-associate learning. Psychonomic Science, 1966, 5, 235-236.

PAIVIO, A., YUILLE, J. C., \& SMYTHE, P. C. Stimulus and response abstractness, imagery, and meaningfulness, and reported mediators in paired-associate learning. Canadian Journal of Psychology, 1966, 20, 362-377.

THORNDIKE, E. L., \& LORGE, I. The teacher's word book of 30,000 words New York: Bureau of Publications, Teachers College, 1944. 